Original Paper http://ajol.info/index.php/ijbcs http://indexmedicus.afro.who.int

\title{
Caractérisation physico-chimique des eaux des aquifères du Continental Intercalaire / Hamadien et du Continentalsiems Terminal de la région de Zinder (Niger)
}

\author{
Rabilou SOULEY MOUSSA ${ }^{1 *}$, Maman Mousbahou MALAM ALMA ${ }^{1}$, \\ Mahaman Sani LAOUALI ${ }^{1}$, Ibrahim NATATOU ${ }^{1}$ et HABOU Issa ${ }^{2}$ \\ ${ }^{1}$ Université Abdou Moumouni de Niamey, Laboratoire d'Analyse des Matériaux, \\ Eau et Environnement, B.P :10662 Niamey, Niger. \\ ${ }^{2}$ Laboratoire d'Analyse des Eaux de la Direction Régionale de l'Hydraulique \\ et de l'Assainissement de Zinder, B.P: 480, Niger. \\ *Auteur correspondant ; E-mail: souleymoussarabilou@yahoo.fr; Tel : (+227) 97930682
}

\section{RESUME}

L'agriculture et l'élevage constituent les principales activités socio-économiques de la population de la région de Zinder (Niger). Toutefois, ces activités présentent des impacts négatifs sur la qualité des eaux souterraines constituant l'une des principales sources d'approvisionnement en eau de cette population. Cette étude a pour objectif de déterminer la qualité physico-chimique et l'origine de la minéralisation des eaux des aquifères du Continental Intercalaire / Hamadien et du Continental Terminal de la région de Zinder. Vingt-huit échantillons ont fait l'objet d'une analyse physico-chimique. Les résultats obtenus ont été traités par la méthode hydrochimique et l'Analyse en Composantes Principales (ACP) avec des logiciels Diagramme et XLSTAT 2016. Les eaux analysées se classent en trois catégories (36\% acides, 32\% neutres et 32\% basiques) et se répartissent en quatre faciès $(53,57 \%$ bicarbonatés sodi-potassiques, $25 \%$ chlorurés sodi-potassiques, $17,85 \%$ chlorurés calci-magnésiens et $3,57 \%$ bicarbonatés calci-magnésiens). L'ACP a permis d'identifier deux mécanismes qui gouvernent la minéralisation des eaux de la région (pluviolessivage de sols par infiltration des minéraux riches en calcium et magnésium et temps de séjour par altération des minéraux). Les teneurs anormales en fluorures de Koundoumawa (3,28 mg.L $\left.\mathrm{L}^{-1}\right)$, Belbedji $\left(1,95 \mathrm{mg} . \mathrm{L}^{-1}\right)$ et Garagoumsa $\left(1,64 \mathrm{mg} . \mathrm{L}^{-1}\right)$ font appel à des traitements de dépollution avant approvisionnement.

(C) 2018 International Formulae Group. All rights reserved.

Mots clés: Eaux souterraines, minéralisation, fluorure, Zinder.

\section{Physicochemical characterization of aquifer waters of the Continental Intercalaire / Hamadien and Continental Terminal in the Zinder region (Niger)}

\begin{abstract}
Agriculture and livestock are the main socio-economic activities of the population of the Zinder region (Niger). However, these activities have negative impacts on groundwater quality, which is one of the main sources of water supply for this population. The aim of this study was to determine the physico-chemical
\end{abstract}


quality and the origin of the mineralization of the aquifer waters of the Continental Intercalaire / Hamadien and the Continental Terminal of the Zinder region. Twenty-eight samples were subjected to physico-chemical analysis. The results obtained were processed by the hydrochemical method and the Principal Components Analysis (PCA) with software Diagram and XLSTAT 2016. The waters analyzed fall into three categories (36\% acids, $32 \%$ neutral and $32 \%$ basic) and are divided into four facies (53.57\% sodi-potassium bicarbonates, $25 \%$ sodium-potassium chlorides, $17.85 \%$ chlorinated calcium-Magnésiens and 3,57\% bicarbonatés calcimagnésiens). The PCA has identified two mechanisms that govern the mineralization of the region's waters (pluviolessivage of soils by infiltration of minerals rich in calcium and magnesium and residence time by mineral alteration). The abnormal fluoride levels of Koundoumawa (3.28 mg.L-1), Belbedji (1.95 mg.L-1) and Garagoumsa (1.64 mg.L-1) use depollution treatments before supply.

(C) 2018 International Formulae Group. All rights reserved.

Keywords: Groundwater, mineralization, fluoride, Zinder.

\section{INTRODUCTION}

Les eaux souterraines constituent l'une des principales sources d'approvisionnement en eau potable de la population dans la plupart des régions du monde (Boubakar, 2010). Cette ressource si fondamentale pour l'existence et l'économie d'un pays ou d'une région doit faire l'objet d'une gestion durable, cohérente et rationnelle car elle est menacée par diverses sources des pollutions d'origine géogénique et anthropique. Les eaux souterraines acquièrent majoritairement leurs compositions chimiques selon la nature géologique des sols traversés, le temps de séjour dans le réservoir et les substances réactives qu'elles pourraient rencontrer lors de l'écoulement (Matini et al., 2009, Yao et al., 2012, Amadou et al., 2014). Cette composition peut être altérée par des substances indésirables voir toxiques issues de l'élevage, des activités agricoles, industrielles, humaines et de la roche encaissante, ce qui rend ces eaux souterraines impropres à la consommation. L'eau participe de façon importante à la régénération de nouvelles cellules et des nouveaux organes chez les êtres humains. Mais quand elle est polluée, elle constitue un vecteur de transmission de plusieurs maladies. Dans le Nord-Ouest et Sud-Ouest de la région de Zinder, l'approvisionnement en eau potable des populations en milieu rural est assuré par des puits et forages. La majeure partie de ces ouvrages, à part quelques analyses physicochimiques réalisées pour s'assurer de la potabilité des eaux après foration et avant livraison aux populations, ne subit aucun contrôle de qualité de manière régulière. L'utilisation intensive et incontrôlée des intrants agricoles et des fumiers pendant la saison des pluies ont un impact négatif sur la qualité de ces eaux. D'où la nécessité de caractériser les eaux souterraines des aquifères du Continental Intercalaire / Hamadien $(\mathrm{CI} / \mathrm{H})$ et du Continental Terminal (CT) de la région de Zinder. Cette étude a pour objectif d'évaluer la qualité physico-chimique des eaux des aquifères du Continental Intercalaire/ Hamadien et du Continental Terminal de la région de Zinder afin de voir leur qualité, les différents mécanismes qui sont à l'origine de la minéralisation de ces eaux et de proposer des solutions aux problèmes de pollution.

\section{MATÉRIEL ET MÉTHODES}

\section{Présentation de la zone d'étude}

L'aquifère du Continental Intercalaire / Hamadien est prédominant dans la partie Nord-Ouest de la région de Zinder (Figure 1). Il est constitué par des bancs d'argiles et des grès ferrugineux (Sandao, 2013). L'aquifère du Continental Terminal est représenté dans le Sud-Ouest de Matamèye et l'Ouest de Magaria (Figure 1). Il est constitué par de grès sidérolithiques moyens à grossiers, reposant en discordance sur le socle (Sandao, 2013). C'est un aquifère peu épais $(50 \mathrm{~m})$, libre et poreux.

\section{Méthodologie}

Dans cette étude, une campagne d'échantillonnage a été réalisée pendant le mois d'août 2016 sur vingt-huit (28) ouvrages 
qui captent les aquifères du $\mathrm{CI} / \mathrm{H}$ et du CT. Les ouvrages échantillonnés sont repartis comme suit :

- Vingt-et-un (21) forages provenant des mini adductions d'eau potable nommés (FA),

- Cinq (5) Puits cimentés nommés (PC),

- Deux (2) forages à Motricité Humaine nommés (FM).

Le choix de ces ouvrages est dicté par le fait qu'ils sont les plus utilisés par les populations rurales. Les coordonnées géographiques des ouvrages ont été obtenues à l'aide d'un GPS (GARMIN GPSMAP64s). Les prélèvements ont été effectués dans des flacons en polyéthylène de $1 \mathrm{~L}$ bien préparés. Les échantillons ont été conservés dans une glacière contenant de la glace puis acheminés directement au laboratoire d'analyse des eaux de la direction régionale de l'hydraulique et de l'assainissement de Zinder. Au cours de cette étude, quatorze (14) paramètres ont été déterminés : le $\mathrm{pH}$, la température $\left(\mathrm{T}^{\circ}\right)$, la conductivité électrique (CE), les ions potassium $\left(\mathrm{K}^{+}\right)$, sodium $\left(\mathrm{Na}^{+}\right)$, calcium $\left(\mathrm{Ca}^{2+}\right)$, magnésium $\left(\mathrm{Mg}^{2+}\right)$, fer total $\left(\mathrm{Fe}^{2+}, \mathrm{Fe}^{3+}\right)$, bicarbonate $\left(\mathrm{HCO}_{3}{ }^{-}\right)$, chlorure $\left(\mathrm{Cl}^{-}\right)$, fluorure $\left(\mathrm{F}^{-}\right)$, nitrate $\left(\mathrm{NO}_{3}^{-}\right)$, nitrite $\left(\mathrm{NO}_{2}^{-}\right)$, et sulfate $\left(\mathrm{SO}_{4}{ }^{2-}\right)$.

Les paramètres physiques $(\mathrm{pH}, \mathrm{CE}$ et $\mathrm{T}^{\circ}$ ) ont été mesurés in situ à l'aide d'un $\mathrm{pH}$ mètre de marque Palintest et d'un conductimètre de marque EUTECH INSTRUMENTS (Cyber Scan com. 110).

Les paramètres chimiques à savoir : $\mathrm{F}^{-}$, $\mathrm{SO}_{4}{ }^{2-}$, fer total, $\mathrm{NO}_{3}{ }^{-}$et $\mathrm{NO}_{2}^{-}$ont été analysés par colorimétrie à l'aide d'un spectrophotomètre DR-2800 de marque Hach, le $\mathrm{K}^{+}$et le $\mathrm{Na}^{+}$par absorption atomique à l'aide d'un spectrophotomètre à flamme de marque JENWAY. Le $\mathrm{Ca}^{2+}$ et le $\mathrm{Mg}^{2+}$ ont été analysés par complexométrie à l'EDTA, le $\mathrm{HCO}_{3}{ }^{-}$par titrimétrie à l'acide sulfurique à l'aide d'un Titrateur Digital de marque Hach et le $\mathrm{Cl}^{-}$par la méthode de Mohr. Toutes ces analyses ont été réalisées suivant le protocole décrit par Jean Rodier (Rodier et al., 2009).

Les coordonnées des ouvrages ont été utilisées pour la réalisation de la carte de répartition spatiale à l'aide du logiciel
ArcGIS9.3 (Figure 1). Les données issues de ces analyses ont été comparées aux valeurs guide de l'OMS pour une eau de consommation.

\section{Analyse statistique}

Les données obtenues ont fait l'objet d'une analyse statistique et d'une analyse hydrochimique. Du point de vue hydrochimique le logiciel Diagramme a été utilisée pour la classification chimique des eaux, basé sur une répartition graphique des cations majeurs $\left(\mathrm{Ca}^{2+}, \mathrm{Mg}^{2+}, \mathrm{Na}^{+}\right.$et $\left.\mathrm{K}^{+}\right)$et anions majeurs $\left(\mathrm{HCO}_{3}^{-}, \mathrm{Cl}^{-}, \mathrm{SO}_{4}{ }^{2-}\right.$ et $\left.\mathrm{NO}_{3}{ }^{-}\right)$ dans le diagramme triangulaire de Piper.

L'analyse statistique a été réalisée sur des variables centrées réduites. Cette analyse a pour but de comprendre les différentes corrélations qui existent entre les différentes variables prises deux à deux, de choisir les différents axes factoriels, la contribution de chaque variable à la constitution des axes factoriels et les différents mécanismes qui interviennent dans la minéralisation des eaux souterraines. En effet, le coefficient de corrélation exprime le niveau de corrélation linéaire entre les variables. Les variables sont corrélées positivement lorsqu'il est proche de 1 et négativement lorsqu'il est proche de -1 . L'analyse descriptive des paramètres physicochimique a concerné les valeurs extrêmes (minimum et maximum), la moyenne (valeur centrale), l'écart- type (paramètre de dispersion). Dans cette étude l'analyse statistique a été réalisée sur 28 échantillons et 14 variables $\left(\mathrm{pH}, \mathrm{T}^{\circ}, \mathrm{CE}, \mathrm{Ca}^{2+}, \mathrm{Mg}^{2+}, \mathrm{Na}^{+}, \mathrm{K}^{+}\right.$, $\mathrm{HCO}_{3}^{-}, \mathrm{Cl}^{-}, \mathrm{SO}_{4}{ }^{2-}, \mathrm{NO}_{3}^{-}, \mathrm{NO}_{2}^{-}, \mathrm{F}^{-}$et le fer total) à l'aide du logiciel XLSTAT 2016. Ainsi le critère de Kaiser a été retenu pour le choix des axes factoriels (seuls les axes dont les valeurs propres sont supérieures à 1 vont être utilisés). Ces logiciels sont très utilisés en hydrochimie pour caractériser l'origine de la minéralisation et les faciès chimiques des eaux et donnent de très bons résultats (Chemseddine et al., 2009; Oga et al., 2009; Kouassi et al., 2010 ; Ahoussi et al., 2010 ; Lasm et al., 2011; Ahoussi et al., 2013; Amadou et al., 2014; Issa et al., 2015 ; Sridhar et al., 2016 ; Loukman et al., 2017). 


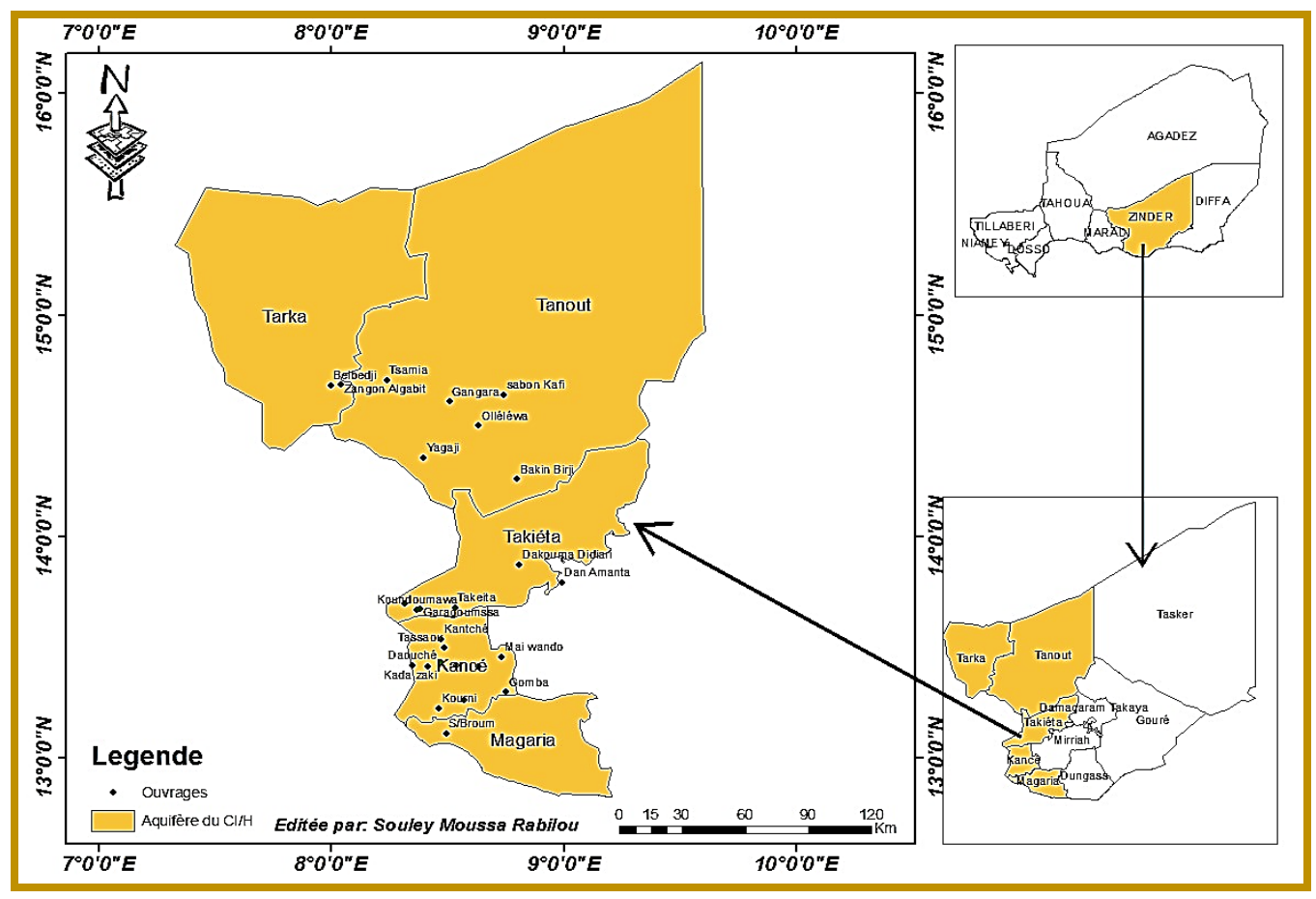

Figure 1 : Localisation de la zone d'étude et des différents ouvrages échantillonnés.

\section{RESULTATS \\ Analyses physico-chimiques}

Les résultats des analyse physicochimiques des eaux des aquifères du $\mathrm{CI} / \mathrm{H}$ et du CT sont présentés dans Tableau 1. Le $\mathrm{pH}$ des eaux analysées varie de 5,6 à 8,96, avec une moyenne de 7,65 et un écart-type de 1,33. Des $\mathrm{pH}$ relativement acides et basiques ont été enregistrés respectivement dans les ouvrages qui captent l'aquifère du $\mathrm{CT}\left(\mathrm{FA}_{16}, \mathrm{FA}_{17}\right.$, $\mathrm{FA}_{18}, \mathrm{PC}_{2}, \mathrm{PC}_{3}, \mathrm{PC}_{4}, \mathrm{FA}_{20}, \mathrm{FA}_{21}$, et $\mathrm{PC}_{5}$ ) et l'aquifère du $\mathrm{CH}\left(\mathrm{FA}_{1}, \mathrm{FA}_{2}, \mathrm{FA}_{3}, \mathrm{FA}_{4}, \mathrm{FA}_{5}\right.$, $\mathrm{FA}_{6}, \mathrm{FA}_{7}, \mathrm{FA}_{8}, \mathrm{FA}_{9}$ et $\left.\mathrm{FA}_{10}\right)$. Les autres ouvrages qui captent l'aquifère du CI présentent des $\mathrm{pH}$ légèrement alcalins. Les eaux analysées se classent en trois catégories : eaux légèrement acides, eaux légèrement neutres et eaux légèrement basiques. La température des eaux varie entre 27,8 et 32,1 ${ }^{\circ} \mathrm{C}$, avec une moyenne de $30,89{ }^{\circ} \mathrm{C}$ et un écart-type de $1,16^{\circ} \mathrm{C}$. Ces eaux présentent des conductivités électriques qui varient de 30 à $1262 \mu \mathrm{S} . \mathrm{cm}^{-1}$, avec une moyenne de 257,19 $\mu$ S.cm ${ }^{-1}$ et un écart-type de $253,96 \mu$ S.cm ${ }^{-1}$. Des faibles conductivités ont été relevées dans certains ouvrages captant l'aquifère du $\mathrm{CT}$ $\left(\mathrm{FA}_{16}, \mathrm{FA}_{17}, \mathrm{FA}_{18}, \mathrm{PC}_{2}, \mathrm{FA}_{20}, \mathrm{FA}_{21}\right.$, et $\left.\mathrm{PC}_{5}\right)$. Une forte conductivité a été enregistrée dans un seul ouvrage qui capte l'aquifère du CI $\left(\mathrm{FA}_{12}\right)$. Concernant les cations majeurs $\left(\mathrm{Ca}^{2+}\right.$, $\mathrm{Mg}^{2+}, \mathrm{Na}^{+}$et $\mathrm{K}^{+}$), les ions $\mathrm{Na}^{+}$sont les plus prédominants et varient entre 0,76 et 175 mg. $\mathrm{L}^{-1}$, avec une moyenne de $51,42 \mathrm{mg} . \mathrm{L}^{-1}$ et écart-type de 44,47 mg. $\mathrm{L}^{-1}$. Ensuite vient les ions $\mathrm{Ca}^{2+}$ avec une variation de 1,6 à 92,8 mg. $\mathrm{L}^{-1}$, une moyenne de $12,57 \mathrm{mg} . \mathrm{L}^{-1}$ et un écart-type de $16,9 \mathrm{mg} \cdot \mathrm{L}^{-1}$. Les ions $\mathrm{K}^{+}$ occupent la troisième position et varient entre 0 et $16 \mathrm{mg} . \mathrm{L}^{-1}$, avec une moyenne de 2,85 $\mathrm{mg} . \mathrm{L}^{-1}$ et un écart-type de $3,34 \mathrm{mg} . \mathrm{L}^{-1}$. Les ions $\mathrm{Mg}^{2+}$ occupent la dernière position et varient de 0 à $15,56 \mathrm{mg} . \mathrm{L}^{-1}$, avec une moyenne de $2,46 \mathrm{mg} . \mathrm{L}^{-1}$ et un écart-type de 3,92 mg. $\mathrm{L}^{-1}$. Concernant les anions majeurs $\left(\mathrm{HCO}_{3}{ }^{-}, \mathrm{Cl}^{-}, \mathrm{SO}_{4}{ }^{2-}\right.$ et $\left.\mathrm{NO}_{3}{ }^{-}\right)$, les ions $\mathrm{HCO}_{3}{ }^{-}$ prédominent et varient de 4,88 à $351,36 \mathrm{mg} . \mathrm{L}^{-}$ 1 , avec une moyenne de $98,55 \mathrm{mg} . \mathrm{L}^{-1}$ et un écart-type de 73,27 mg. $\mathrm{L}^{-1}$. Ensuite vient les ions $\mathrm{Cl}^{-}$avec une variation de 11,36 à 284 mg. $\mathrm{L}^{-1}$, une moyenne de $37,47 \mathrm{mg} . \mathrm{L}^{-1}$ et un 
écart-type de $55,01 \mathrm{mg} \cdot \mathrm{L}^{-1}$. Les ions $\mathrm{SO}_{4}{ }^{2-}$ occupent la troisième position et varient de 0 à $90 \mathrm{mg} . \mathrm{L}^{-1}$, avec une moyenne de $15,17 \mathrm{mg} . \mathrm{L}^{-1}$ et un écart-type de 25,39 mg. $\mathrm{L}^{-1}$. En fin, les ions nitrates occupent la dernière position et varient entre 1,32 et $58,96 \mathrm{mg} \cdot \mathrm{L}^{-1}$, avec une moyenne de $9,71 \mathrm{mg} . \mathrm{L}^{-1}$ et un écart-type de $12,06 \mathrm{mg} \cdot \mathrm{L}^{-1}$. Une teneur légèrement élevée en nitrate a été enregistrée dans l'ouvrage $\mathrm{PC}_{4}$. Pour les ions mineurs $\left(\mathrm{NO}_{2}^{-}, \mathrm{F}^{-}\right.$et fer total), ils sont dans l'ensemble moins abondant dans les eaux analysées, mais des fortes teneurs en fluorures ont été enregistrées dans certains ouvrages captant les aquifères du $\mathrm{CI} / \mathrm{H}\left(\mathrm{FA}_{1}\right.$, $\mathrm{FM}_{1}$ et $\mathrm{FM}_{2}$ ). La Figure 2 présente la répartition des ions fluorures dans la zone d'étude. Il ressort de cette Figure 2 que 11\% des ouvrages $\left(\mathrm{FA}_{1}, \mathrm{FM}_{1}\right.$ et $\left.\mathrm{FM}_{2}\right)$ ont des teneurs en fluorures qui dépassent la valeur guide de l'OMS (1,5 mg. $\left.\mathrm{L}^{-1}\right), 18 \%$ ont des teneurs comprises entre 0,7 et $1,5 \mathrm{mg} . \mathrm{L}^{-1}$ et $71 \%$ ont des teneurs comprises entre 0 et 0,7 $\mathrm{mg} \cdot \mathrm{L}^{-1}$. De manière générale, les teneurs enregistrés en fluorure dépassant la valeur guide de l'OMS, peuvent provoquer des risques sanitaires pour la consommation. Mais celles enregistrées comprises entre 0,7 et 1,5 $\mathrm{mg} . \mathrm{L}^{-1}$, peuvent aussi provoquer des risques sanitaires surtout en zone tempérée. Celles enregistrées inférieurs à $0,7 \mathrm{mg} . \mathrm{L}^{-1}$, sont bénéfiques et protègent les dents contre la carie dentaire.

\section{Faciès hydrochimique}

L'analyse des résultats chimiques (cation et anion majeur) par la méthode hydrochimique qui utilise le digramme triangulaire de Piper montre que les eaux souterraines du $\mathrm{CI} / \mathrm{H}$ et du $\mathrm{CT}$ se répartissent en quatre types de faciès (Figure3) :

- Faciès bicarbonaté sodique et potassique qui ne contient que des forages $\left(\mathrm{FA}_{1}, \mathrm{FA}_{2}\right.$, $\mathrm{FA}_{4}, \mathrm{FA}_{5}, \mathrm{FA}_{6}, \mathrm{FA}_{7}, \mathrm{FA}_{8}, \mathrm{FA}_{9}, \mathrm{FA}_{10}, \mathrm{FA}_{11}$, $\mathrm{FA}_{14}, \mathrm{FA}_{16}, \mathrm{FA}_{19}, \mathrm{FA}_{21}, \mathrm{FM}_{2}$ );

- Faciès chloruré sodique et potassique qui contient des forages $\left(\mathrm{FA}_{12}, \mathrm{FA}_{13}, \mathrm{FA}_{17}\right.$, $\left.\mathrm{FA}_{18}, \mathrm{FA}_{20}, \mathrm{FM}_{1}\right)$ et un seul puits $\left(\mathrm{PC}_{3}\right)$;

- Faciès chloruré calcique et magnésien qui contient des puits $\left(\mathrm{PC}_{1}, \mathrm{PC}_{2}, \mathrm{PC}_{4}, \mathrm{PC}_{5}\right)$ et un seul forage $\left(\mathrm{FA}_{15}\right)$;
- Faciès bicarbonaté calcique et magnésien constitué d'un seul forage $\left(\mathrm{FA}_{3}\right)$.

Dans le triangle des cations, $78,57 \%$ des eaux se concentrent dans le pôle sodipotassique, $7,14 \%$ dans le pôle calcique, $14,28 \%$ dans la zone où aucun cation ne domine. De même dans le triangle des anions, $60,71 \%$ des eaux se concentrent dans le pôle bicarbonaté et $39,28 \%$ dans le pôle chlorurénitraté. De manière générale, les espèces dominantes de ces eaux sont le bicarbonate et le sodium. Le faciès bicarbonaté sodipotassique reste le plus abondant.

\section{Analyse en composantes principales (ACP)}

L'analyse en composantes principales normées a été réalisée sur 28 échantillons et 14 paramètres $\left(\mathrm{pH}, \mathrm{T}^{\circ}, \mathrm{CE}, \mathrm{F}^{-}, \mathrm{SO}_{4}{ }^{2-}\right.$, fer total, $\left.\mathrm{NO}_{3}^{-}, \mathrm{NO}_{2}^{-}, \mathrm{K}^{+}, \mathrm{Na}^{+}, \mathrm{Ca}^{2+}, \mathrm{Mg}^{2+}, \mathrm{HCO}_{3}^{-}, \mathrm{Cl}^{-}\right)$. Cette analyse permet de calculer les valeurs propres, les variances exprimées pour chaque axe factoriel, leur cumul, les coefficients de corrélation entre tous les paramètres pris deux à deux, ainsi que la statistique descriptive et la participation de chaque paramètre à la constitution des axes factoriels. Les résultats de cette analyse sont présentés dans les Tableaux suivants: Tableau 2 pour la statistique descriptive, Tableau 3 pour la matrice de corrélation, Tableau 4 pour les valeurs propres et Tableau 5 pour les cosinus carrés et les paramètres. Le Tableau 2 résumant l'analyse descriptive réalisée sur les paramètres physico-chimiques montre que la majeure partie des écart-types est supérieure aux moyennes de différents paramètres, le rapport écart-type à la moyenne ou coefficient de variation $(\mathrm{Cv})$ est supérieur à 0,25 dans l'ensemble de paramètres excepté le $\mathrm{pH}$ et la température, ce qui montre une hétérogénéité des eaux de ces deux aquifères. Certains paramètres présentent des valeurs maximales qui sont supérieures aux valeurs guide de l'OMS et d'autres présentent des valeurs minimales qui n'atteignent pas celles de l'OMS. Mais, les valeurs moyennes dans tous les paramètres restent dans la gamme des valeurs guides de l'OMS. La matrice de corrélation (Tableau 3) montre des bonnes corrélations entre le $\mathrm{Ca}^{2+}$ et le $\mathrm{Cl}^{-}(0,903)$, le 
$\mathrm{Na}^{+}$et les éléments $\mathrm{HCO}_{3}{ }^{-}(0,830), \mathrm{SO}_{4}{ }^{2-}$ $(0,692), \mathrm{Cl}^{-}(0,648)$, la $\mathrm{CE}$ et les éléments $\mathrm{Ca}^{2+}$ $(0,749), \mathrm{SO}_{4}^{2-}(0,735), \mathrm{Cl}^{-}(0,688)$, le $\mathrm{Mg}^{2+}$ et le $\mathrm{K}^{+}(0,759)$ et enfin le $\mathrm{pH}$ et les éléments $\mathrm{T}^{\circ}$ $(0,732), \mathrm{HCO}_{3}^{-}(0,660)$. Aucune corrélation n'est observée entre les éléments suivants : $\mathrm{F}^{-}$, fer total, $\mathrm{NO}_{3}{ }^{-}, \mathrm{NO}_{2}{ }^{-}$et les autres paramètres. Cette matrice montre clairement les différents liens qui existent entre les différentes variables et leur évolution dans le milieu souterrain. Le Tableau 4 montre que les trois premiers axes expriment $65,56 \%$ de la variance totale dont $33,05 \%$ pour le premier axe, $21,22 \%$ pour le deuxième et $11,3 \%$ pour le troisième. La poursuite de l'analyse jusqu'à trois axes factoriels est due au faite que les deux premiers axes ne donnent pas une variance totale suffisante pour fournir des renseignements sur les différentes interactions hydrogéochimiques qui se déroulent dans les aquifères du $\mathrm{CI} / \mathrm{H}$ et du $\mathrm{CT}$ de la région de Zinder. Les trois axes sélectionnés présentent des valeurs propres supérieures à 1 (donc ils remplissent le critère de Kaiser). Le Tableau 5 montre les valeurs des différentes variables participant à la constitution des différents axes factoriels. Il ressort de ce tableau, que les valeurs représentées en gras correspondent aux valeurs des variables pour lequel le cosinus carré est le plus grand pour chaque axe factoriel. Plus le cosinus carré est élevé plus le paramètre est lié à l'axe factoriel et plus le cosinus carré tend vers zéro moins le paramètre est lié à l'axe factoriel. L'importance du cosinus carré est de voir réellement l'angle formé par le vecteur de ce point et le plan de projection de la variable pour évaluer la qualité de la projection de ce paramètre sur les différents axes. Plus de $42 \%$ des variables participent à la constitution de l'axe factoriel $\mathrm{F} 1$ dont un cation majeur $\left(\mathrm{Na}^{+}\right)$, trois anions majeurs $\left(\mathrm{HCO}_{3}^{-}, \mathrm{Cl}^{-}\right.$et $\left.\mathrm{SO}_{4}^{2-}\right)$, le $\mathrm{pH}$ et la CE. Donc ce facteur rassemble le maximum des ions participant à la minéralisation des eaux analysées. Le facteur F2 présente $21 \%$ des variables qui participent à sa constitution, dont deux cations majeurs $\left(\mathrm{Ca}^{2+}\right.$ et $\left.\mathrm{Mg}^{2+}\right)$ et un anion majeur $\left(\mathrm{NO}_{3}^{-}\right) . \mathrm{Ce}$ facteur regroupe les alcalinoterreux et l'ion ayant une origine principalement anthropique. Le facteur F3 présente $14 \%$ des variables qui participent à sa constitution, le $\mathrm{K}^{+}$et le Fer total. En effet, deux paramètres proches sur l'espace réduit pourraient avoir des comportements différents par rapport aux axes factoriels.

\section{Analyse de l'espace des variables des plans factoriels F1 x F2 et F1 x F3}

La Figure 4 résume les espaces réduits de trois premières composantes principales.

Le facteur $\mathrm{F} 1$ est défini par $\mathrm{CE}, \mathrm{Cl}^{-}$, $\mathrm{pH}, \mathrm{SO}_{4}^{2-}, \mathrm{Na}^{+}$et $\mathrm{HCO}_{3}^{-}$(Figure 4a). Le regroupement de ces éléments suggère la provenance d'une source commune et d'un mécanisme identique de la mise en solution de ces éléments. Compte tenu des corrélations existantes entre ces différents éléments, ce regroupement traduirait une minéralisation des eaux liées aux contacts eau-roche pour une longue durée de mise en solution. Ce facteur exprime l'acquisition de la majeure partie des ions et de la CE ce qui traduirait le phénomène temps de séjours des eaux dans les réservoirs. Le facteur F2 (Figure4a) est déterminé par $\mathrm{Ca}^{2+}, \mathrm{Mg}^{2+}, \mathrm{NO}_{3}^{-}$, le rassemblement de ces éléments autour de cet axe montre qu'ils seraient mis en solution par le même phénomène, mais, leurs positions dans le pôle opposé montrent qu'ils sont mis en solution par des mécanismes différents. Les $\mathrm{Ca}^{2+}$ et $\mathrm{Mg}^{2+}$ seraient issus de l'infiltration par lessivages des minéraux riches en calcium et magnésium et les nitrates sont issus des apports superficiels qui témoignent une pollution anthropique. Ce facteur traduit le phénomène de pluviolessivage des sols et de la vulnérabilité de la nappe à la pollution. Le facteur F3 (Figure 4b) est représentatif du $\mathrm{K}^{+}$ et du Fer, mais la position de ces éléments de part et d'autre de cet axe montre qu'ils sont mis en solution par des mécanismes différents. Le $\mathrm{Fe}$ total proviendrait du lessivage des horizons superficiels et de l'hydrolyse acide des minéraux ferromagnésien et le $\mathrm{K}^{+}$ proviendrait de l'altération des minéraux renfermant le potassium tel que l'orthose. 


\section{R. S. MOUSSA et al. / Int. J. Biol. Chem. Sci. 12(5): 2395-2411, 2018}

Tableau 1: Données physico-chimiques en mg. $\mathrm{L}^{-1}$ des eaux des aquifères du CI/H et du CT de la région de Zinder (août 2016) sauf (CE, pH, T).

\begin{tabular}{|c|c|c|c|c|c|c|c|c|c|c|c|c|c|c|c|}
\hline Nom & Ouvrage & $\mathbf{T}{ }^{\circ} \mathbf{C}$ & $\mathbf{p H}$ & $\mathrm{CE} \mu \mathrm{S} / \mathrm{cm}$ & $\mathrm{Ca}^{2+}$ & $\mathrm{Mg}^{2+}$ & $\mathrm{Na}^{+}$ & $\mathbf{K}^{+}$ & Fer total & $\mathrm{HCO}_{3}^{-}$ & $\mathrm{Cl}^{-}$ & $\mathrm{SO}_{4}{ }^{2-}$ & $\mathrm{NO}_{3}^{-}$ & $\mathbf{F}^{-}$ & $\mathrm{NO}_{2}^{-}$ \\
\hline Belbedji & $\mathrm{FA}_{1}$ & 31,9 & 8,88 & 225 & 6,4 & 0 & 85,03 & 0,75 & 0 & 175,68 & 15,62 & 32 & 3,08 & 1,95 & 0,0297 \\
\hline Gangara & $\mathrm{FA}_{2}$ & 32,1 & 8,96 & 397 & 4,8 & 1,95 & 67 & 0 & 0,03 & 131,76 & 14,2 & 35 & 1,32 & 1,12 & 0,0264 \\
\hline Bakin Birji & $\mathrm{FA}_{3}$ & 32,1 & 8,71 & 214 & 24 & 2,92 & 18,2 & 2,25 & 0 & 112,24 & 12,78 & 0 & 5,28 & 0 & 0,033 \\
\hline Tsamia & $\mathrm{FA}_{5}$ & 32,1 & 8,84 & 286 & 8 & 0 & 71,3 & 0,75 & 0,01 & 131,76 & 26,98 & 27 & 2,2 & 0 & 0,0264 \\
\hline Olléléwa & $\mathrm{FA}_{6}$ & 32 & 8,76 & 302 & 3,2 & 0 & 60,03 & 0 & 0,02 & 122 & 25,56 & 0 & 3,08 & 0,96 & 0,0231 \\
\hline Zangon Algabit & $\mathrm{FA}_{7}$ & 31,5 & 8,62 & 189,2 & 8 & 0 & 41,1 & 3,75 & 0,01 & 87,84 & 17,04 & 15 & 3,08 & 0,02 & 0,0165 \\
\hline Chirwa & $\mathrm{FA}_{8}$ & 31,6 & 8,9 & 431 & 1,6 & 0,98 & 91,34 & 0 & 0 & 136,64 & 15,62 & 68 & 2,2 & 0 & 0,0231 \\
\hline FalenKo & $\mathrm{FA}_{9}$ & 31,6 & 8,82 & 253 & 4,8 & 0 & 54,1 & 0,75 & 0,01 & 122 & 14,2 & 7 & 3,96 & 0,67 & 0,0231 \\
\hline sabon Kafi & $\mathrm{FA}_{10}$ & 31,6 & 8,78 & 446 & 3,2 & 0 & 101 & 0 & 0,02 & 151,28 & 12,78 & 80 & 2,2 & 0,62 & 0,0297 \\
\hline Tassaou & $\mathrm{FA}_{11}$ & 29,9 & 7,64 & 140,4 & 9,6 & 3,89 & 148,94 & 4,75 & 0,02 & 351,36 & 56,8 & 0 & 2,2 & 0,5 & 0,0231 \\
\hline Kantché & $\mathrm{PC}_{1}$ & 30,4 & 7,64 & 756 & 20,8 & 15,56 & 3,7 & 16 & 0,02 & 87,84 & 29,82 & 9 & 25,96 & 0,91 & 0,0132 \\
\hline Dan Amanta & $\mathrm{FA}_{12}$ & 31,3 & 7,52 & 1262 & 92,8 & 8,75 & 175 & 6,05 & 0,42 & 192,76 & 284 & 90 & 4,4 & 0,17 & 0,0429 \\
\hline Dakouma Didiari & $\mathrm{FA}_{13}$ & 31,7 & 7,05 & 198 & 20,8 & 0,98 & 76 & 5,75 & 0,01 & 92,72 & 103,66 & 0 & 7,48 & 0,25 & 0,0594 \\
\hline Garagoumsa & $\mathrm{FM}_{1}$ & 30,9 & 7,12 & 221 & 17,6 & 0 & 102,36 & 5,05 & 0,02 & 85,4 & 122,12 & 21 & 11 & 1,64 & 0,0165 \\
\hline Koundoumawa & $\mathrm{FM}_{2}$ & 31,2 & 7,24 & 358 & 9,6 & 1,95 & 70,6 & 3,75 & 0,03 & 139,08 & 28,4 & 30 & 6,16 & 3,28 & 0,0429 \\
\hline Takeita & $\mathrm{FA}_{15}$ & 30,6 & 6,7 & 201 & 14,4 & 12,64 & 20,7 & 5 & 0,01 & 82,96 & 48,28 & 0 & 3,96 & 0,06 & 0,0264 \\
\hline Daouché & $\mathrm{FA}_{16}$ & 27,9 & 6,08 & 51,2 & 8 & 0 & 13,77 & 3,25 & 0,01 & 34,16 & 14,2 & 0 & 7,48 & 0,04 & 0,0363 \\
\hline Yaouri & $\mathrm{FA}_{17}$ & 27,8 & 5,27 & 35,2 & 3,2 & 0 & 8,9 & 1 & 0,01 & 9,76 & 11,36 & 0 & 5,72 & 0 & 0,0264 \\
\hline Kourni & $\mathrm{FA}_{18}$ & 30,1 & 5,33 & 45,2 & 3,2 & 0,98 & 11 & 0 & 0,85 & 4,88 & 12,78 & 0 & 17,16 & 0 & 0,0396 \\
\hline Kada zaki & $\mathrm{PC}_{2}$ & 29,5 & 5,8 & 64 & 12,8 & 2,92 & 0,76 & 1,5 & 0,03 & 19,52 & 12,78 & 0 & 16,72 & 0,42 & 0,033 \\
\hline Matamey CES & $\mathrm{PC}_{3}$ & 29,9 & 5,85 & 107 & 11,2 & 0,98 & 15,5 & 1,5 & 0,08 & 9,76 & 25,56 & 0 & 29,04 & 0,61 & 0,0429 \\
\hline Godo Haoussa & $\mathrm{FA}_{19}$ & 30,7 & 6,65 & 113 & 6,4 & 1,95 & 22,5 & 6,05 & 0,01 & 58,56 & 19,88 & 1 & 4,4 & 0,45 & 0,0198 \\
\hline Mai wando & $\mathrm{PC}_{4}$ & 29,6 & 5,9 & 225 & 20,8 & 1,95 & 20,13 & 1,5 & 0,01 & 34,16 & 21,3 & 0 & 58,96 & 0,03 & 0,0297 \\
\hline S/Broum & $\mathrm{FA}_{20}$ & 31,1 & 5,18 & 30 & 6,4 & 0 & 12,75 & 0 & 0,03 & 24,4 & 12,78 & 0 & 7,04 & 0 & 0,0264 \\
\hline Gomba & $\mathrm{FA}_{21}$ & 30,8 & 6,21 & 42,1 & 9,6 & 0 & 28,32 & 0,75 & 0,04 & 73,2 & 17,04 & 0 & 3,08 & 0 & 0,0231 \\
\hline Doungou & $\mathrm{PC}_{5}$ & 31,5 & 6,1 & 82,5 & 8 & 6,81 & 7,1 & 4 & 0,08 & 19,52 & 28,4 & 0 & 15,4 & 0,03 & 0,3003 \\
\hline
\end{tabular}




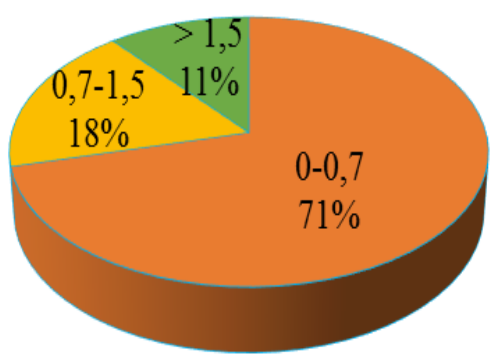

Figure 2 : Répartition des ions fluorures dans la zone d'étude.

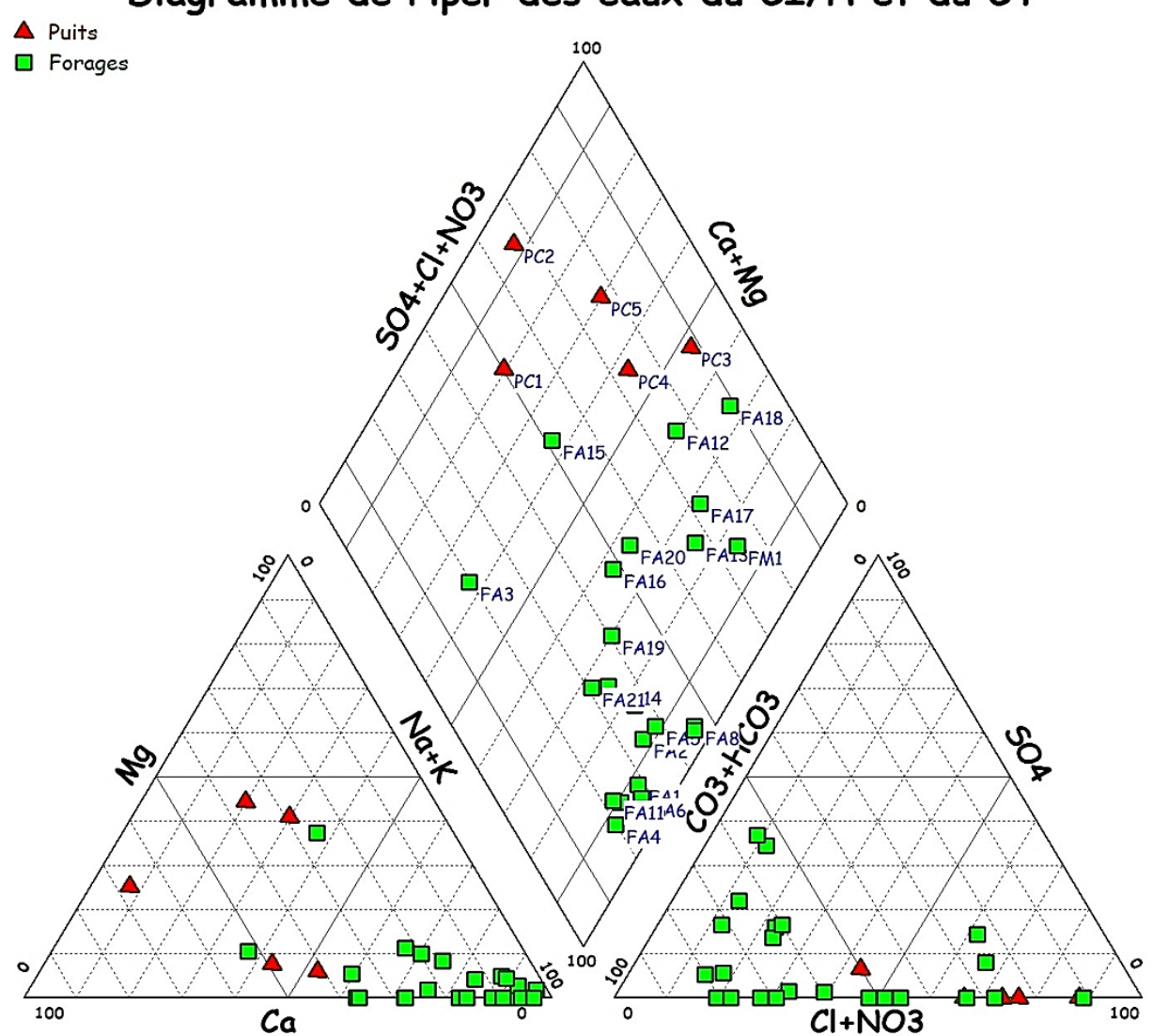

Figure 3 : Diagramme de Piper des eaux du CI/H et du CT de la région de Zinder. 
R. S. MOUSSA et al. / Int. J. Biol. Chem. Sci. 12(5): 2395-2411, 2018

Tableau 2 : Statistique descriptive des résultats physico-chimique des aquifères du CI/H et du CT.

\begin{tabular}{|c|c|c|c|c|c|c|c|c|}
\hline Paramètres physico-chimiques & $\begin{array}{l}\text { Unité de } \\
\text { mesure }\end{array}$ & $\begin{array}{l}\text { Valeurs Guide } \\
\text { OMS } 2011\end{array}$ & Nombre d'échantillon & Minimum & Maximum & Moyenne & $\begin{array}{l}\text { Ecart- } \\
\text { type }\end{array}$ & $\mathrm{Cv}$ \\
\hline $\mathrm{T}^{\circ}$ & ${ }^{\circ} \mathrm{C}$ & & 28 & 27,800 & 32,100 & 30,893 & 1,166 & 0,03774318 \\
\hline $\mathrm{pH}$ & & $6,5-8,5$ & 28 & 5,180 & 8,960 & 7,351 & 1,337 & 0,18188002 \\
\hline $\mathrm{CE}$ & $\mu \mathrm{S} . \mathrm{cm}^{-1}$ & 400 & 28 & 30,000 & 1262,000 & 257,193 & 253,967 & 0,98745689 \\
\hline $\mathrm{Ca}^{2+}$ & $\operatorname{mg} \cdot \mathrm{L}^{-1}$ & ND & 28 & 1,600 & 92,800 & 12,571 & 16,903 & 1,34460266 \\
\hline $\mathrm{Mg}^{2+}$ & $\mathrm{mg} \cdot \mathrm{L}^{-1}$ & ND & 28 & 0,000 & 15,560 & 2,468 & 3,920 & 1,58833063 \\
\hline $\mathrm{Na}^{+}$ & $\operatorname{mg} . \mathrm{L}^{-1}$ & 200 & 28 & 0,760 & 175,000 & 51,421 & 44,478 & 0,86497734 \\
\hline $\mathrm{K}^{+}$ & $\operatorname{mg} . \mathrm{L}^{-1}$ & 12 & 28 & 0,000 & 16,000 & 2,854 & 3,348 & 1,1730904 \\
\hline Fer total & $\operatorname{mg} . \mathrm{L}^{-1}$ & 0,3 & 28 & 0,000 & 0,850 & 0,065 & 0,172 & 2,64615385 \\
\hline $\mathrm{HCO}_{3}{ }^{-}$ & $\mathrm{mg} \cdot \mathrm{L}^{-1}$ & ND & 28 & 4,880 & 351,360 & 98,559 & 73,276 & 0,74347345 \\
\hline $\mathrm{Cl}^{-}$ & $\operatorname{mg} \cdot \mathrm{L}^{-1}$ & 250 & 28 & 11,360 & 284,000 & 37,478 & 55,017 & 1,46798122 \\
\hline $\mathrm{SO}_{4}{ }^{2-}$ & $\mathrm{mg} . \mathrm{L}^{-1}$ & 250 & 28 & 0,000 & 90,000 & 15,179 & 25,398 & 1,67323276 \\
\hline $\mathrm{NO}_{3}^{-}$ & $\mathrm{mg} \cdot \mathrm{L}^{-1}$ & 50 & 28 & 1,320 & 58,960 & 9,271 & 12,069 & 1,30180132 \\
\hline $\mathrm{F}^{-}$ & $\mathrm{mg} \cdot \mathrm{L}^{-1}$ & 1,5 & 28 & 0,000 & 3,280 & 0,580 & 0,770 & 1,32758621 \\
\hline $\mathrm{NO}_{2}^{-}$ & $\mathrm{mg} \cdot \mathrm{L}^{-1}$ & 3 & 28 & 0,010 & 0,300 & 0,038 & 0,052 & 1,36842105 \\
\hline
\end{tabular}


Tableau 3: Matrice de corrélation entre les différentes variables prises deux à deux.

\begin{tabular}{|c|c|c|c|c|c|c|c|c|c|c|c|c|c|c|}
\hline Variables & $\mathbf{T}^{\circ}$ & pH & $\mathrm{CE}$ & $\mathrm{Ca}^{2+}$ & $\mathrm{Mg}^{2+}$ & $\mathbf{N a}^{+}$ & $\mathbf{K}^{+}$ & Fer total & $\mathrm{HCO}_{3}{ }^{-}$ & $\mathrm{Cl}^{-}$ & $\mathrm{SO}_{4}{ }^{2-}$ & $\mathrm{NO}_{3}^{-}$ & $\mathbf{F}^{-}$ & $\mathrm{NO}_{2}^{-}$ \\
\hline $\mathbf{T}^{\circ}$ & 1 & & & & & & & & & & & & & \\
\hline pH & 0,732 & 1 & & & & & & & & & & & & \\
\hline $\mathrm{CE}$ & 0,317 & 0,418 & 1 & & & & & & & & & & & \\
\hline $\mathrm{Ca}^{2+}$ & 0,040 & $-0,033$ & 0,749 & 1 & & & & & & & & & & \\
\hline $\mathrm{Mg}^{2+}$ & $-0,043$ & $-0,065$ & 0,494 & 0,445 & 1 & & & & & & & & & \\
\hline $\mathrm{Na}^{+}$ & 0,376 & 0,517 & 0,586 & 0,449 & $-0,059$ & 1 & & & & & & & & \\
\hline $\mathbf{K}^{+}$ & $-0,112$ & $-0,042$ & 0,405 & 0,376 & 0,759 & $-0,007$ & 1 & & & & & & & \\
\hline Fer total & $-0,100$ & $-0,305$ & 0,174 & 0,306 & 0,082 & 0,050 & $-0,070$ & 1 & & & & & & \\
\hline $\mathrm{HCO}_{3}^{-}$ & 0,400 & 0,660 & 0,427 & 0,198 & 0,079 & $\mathbf{0 , 8 3 0}$ & 0,086 & $-0,153$ & 1 & & & & & \\
\hline Cl- & 0,095 & 0,003 & 0,688 & 0,903 & 0,323 & 0,648 & 0,347 & 0,304 & 0,302 & 1 & & & & \\
\hline $\mathrm{SO}_{4}{ }^{2-}$ & 0,348 & 0,455 & 0,735 & 0,432 & 0,033 & 0,692 & $-0,077$ & 0,128 & 0,419 & 0,469 & 1 & & & \\
\hline $\mathrm{NO}_{3}{ }^{-}$ & $-0,388$ & $-0,470$ & $-0,051$ & 0,105 & 0,185 & $-0,388$ & 0,168 & 0,111 & $-0,444$ & $-0,068$ & $-0,272$ & 1 & & \\
\hline $\mathbf{F}^{-}$ & 0,262 & 0,314 & 0,152 & $-0,109$ & $-0,057$ & 0,269 & 0,125 & $-0,177$ & 0,308 & $-0,001$ & 0,147 & $-0,127$ & 1 & \\
\hline $\mathrm{NO}_{2}^{-}$ & 0,082 & $-0,238$ & $-0,120$ & 0,010 & 0,189 & $-0,171$ & 0,032 & 0,082 & $-0,230$ & 0,028 & $-0,095$ & 0,122 & $-0,146$ & 1 \\
\hline
\end{tabular}


Tableau 4: Valeurs propres et pourcentages exprimés pour les axes principaux.

\begin{tabular}{lccc}
\hline & F1 & F2 & F3 \\
\hline Valeur propre & 4,627 & 2,971 & 1,582 \\
Variabilité (\%) & 33,047 & 21,223 & 11,297 \\
\% cumulé & 33,047 & 54,270 & 65,567 \\
\hline
\end{tabular}

Tableau 5: Cosinus carrés des variables.

\begin{tabular}{lccc}
\hline Variables & $\mathbf{F 1}$ & $\mathbf{F 2}$ & $\mathbf{F 3}$ \\
\hline $\mathrm{T}^{\circ}$ & 0,268 & 0,213 & 0,012 \\
$\mathrm{pH}$ & $\mathbf{0 , 3 9 1}$ & 0,367 & 0,062 \\
$\mathrm{CE}$ & $\mathbf{0 , 7 4 3}$ & 0,113 & 0,003 \\
$\mathrm{Ca}^{2+}$ & 0,413 & $\mathbf{0 , 4 2 0}$ & 0,035 \\
$\mathrm{Mg}^{2+}$ & 0,075 & $\mathbf{0 , 4 3 8}$ & 0,294 \\
$\mathrm{Na}^{+}$ & $\mathbf{0 , 7 6 0}$ & 0,035 & 0,050 \\
$\mathrm{~K}^{+}$ & 0,065 & 0,337 & $\mathbf{0 , 4 5 8}$ \\
$\mathrm{Fer} \mathrm{total}^{-}$ & 0,005 & 0,180 & $\mathbf{0 , 3 4 7}$ \\
$\mathrm{HCO}_{3}{ }^{-}$ & $\mathbf{0 , 5 5 6}$ & 0,127 & 0,019 \\
$\mathrm{Cl}^{-}$ & $\mathbf{0 , 5 1 1}$ & 0,271 & 0,059 \\
$\mathrm{SO}_{4}{ }^{2-}$ & $\mathbf{0 , 5 9 0}$ & 0,005 & 0,093 \\
$\mathrm{NO}_{3}{ }^{-}$ & 0,139 & $\mathbf{0 , 2 9 4}$ & 0,006 \\
$\mathrm{~F}^{-}$ & 0,083 & 0,108 & 0,145 \\
$\mathrm{NO}_{2}{ }^{-}$ & 0,027 & 0,065 & 0,000 \\
\hline & & & \\
\hline
\end{tabular}



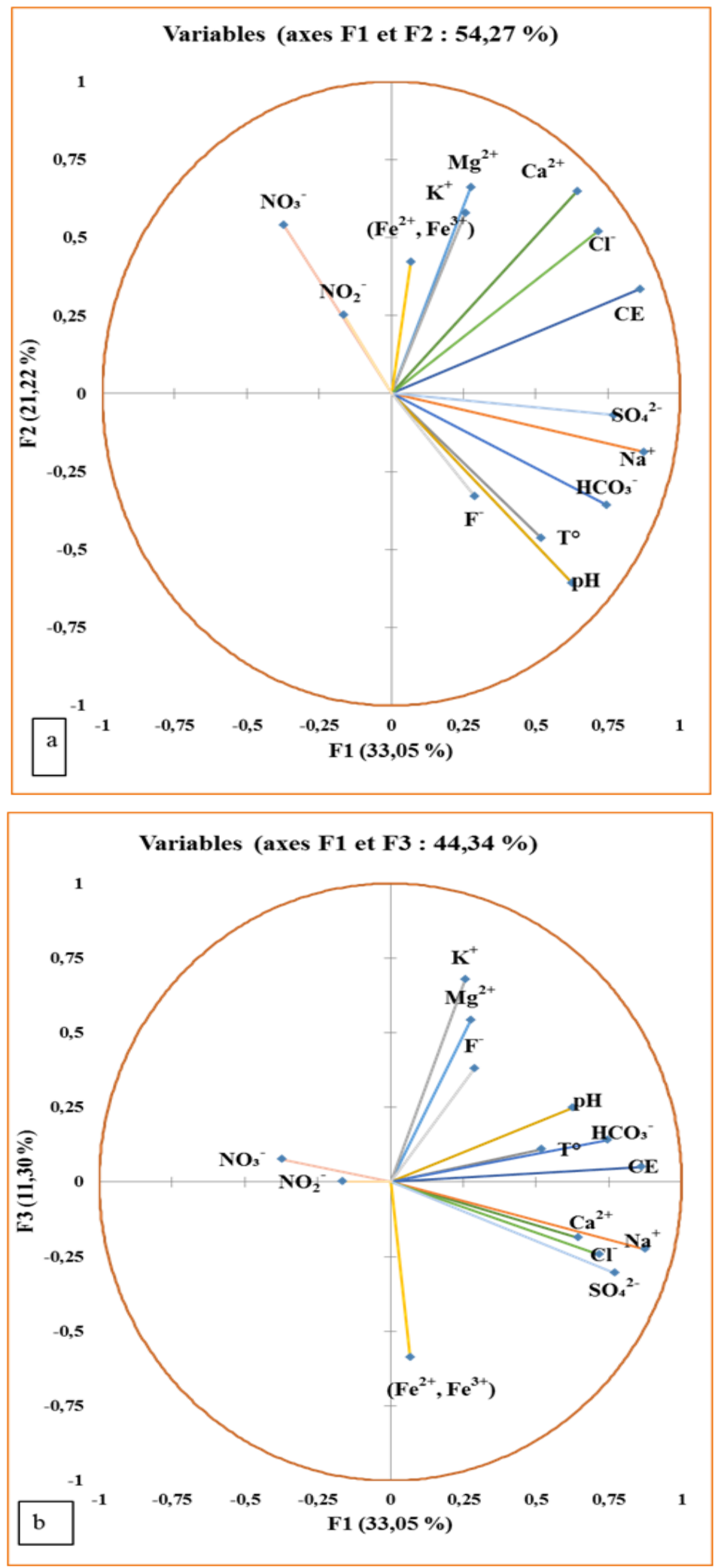

Figure 4 : Espace des variables des plans factoriels a) F1 x F2 et b) F1 x F3. 


\section{DISCUSSION}

\section{Analyses physico-chimiques}

L'étude des paramètres physicochimiques des eaux des aquifères du $\mathrm{CI} / \mathrm{H}$ et du CT montre que ces eaux présentent, dans l'ensemble, des valeurs qui sont conformes aux valeurs guide recommandées par l'OMS (OMS, 2011) pour une eau de consommation. Cependant, des fortes teneurs en fluorures ont été enregistrées respectivement dans les ouvrages $\mathrm{FM}_{2}\left(3,28 \mathrm{mg} . \mathrm{L}^{-1}\right), \mathrm{FA}_{1}\left(1,95 \mathrm{mg} . \mathrm{L}^{-}\right.$ $\left.{ }^{1}\right)$ et $\mathrm{FM}_{1}\left(1,64 \mathrm{mg} \cdot \mathrm{L}^{-1}\right)$ qui dépasse la valeur guide de l'OMS $\left(1,5 \mathrm{mg} \cdot \mathrm{L}^{-1}\right)$. Ces teneurs en fluorure pourraient être dues à la formation géologique de la région, car les grès du Complexe $\mathrm{CI} / \mathrm{H}$ datent du Crétacé supérieur et sont constitués des sédiments détritiques. Dans ce genre de formation, l'apport des ions fluorures par les minéraux tels que la fluorine et la fluoro-apatite est fréquent comme l'a indiqué Malcuit (2012). En effet, les ions fluorures dérivent principalement de la dissolution des minéraux naturels dans les roches et les sols avec lesquels l'eau interagit (Ravindra et Garg, 2007). Les résultats de cette étude sont en accord avec ceux trouvés par Issa et al. (2015) dans la région de Zinder au Niger. Des teneurs en fluorures en dessous et au-dessus des valeurs obtenus dans cette étude ont été rapportées respectivement dans la région de Tahoua au Niger par Amadou et al. (2014) et dans la région du Sahara Septentrional en Algérie par Tabouche et Achour. (2004). Dans les ouvrages qui captent l'aquifère du complexe $\mathrm{CH}$ d'une part et du $\mathrm{CT}$, des $\mathrm{pH}$ acides et des faibles conductivités ont été enregistrés. Les caractéristiques lithologiques de cet aquifère (constitué des niveaux des grès ferrugineux) militent en faveur de la réaction de précipitation de la sidérite $\left(\mathrm{FeCO}_{3}\right)$ suite à la dissolution de l'oxyde de fer (Sandao, 2013). Ces faibles valeurs de $\mathrm{pH}$ pourraient être dues à la réaction chimique consommatrice des ions $\mathrm{HCO}_{3}{ }^{-}$par les ions $\mathrm{Fe}^{2+}$ libérés par l'oxyde de fer en milieu aqueux pour former les $\mathrm{FeCO}_{3}$ et $\mathrm{H}^{+}$. Donc la libération des ions $\mathrm{H}^{+}$entraînera la diminution $\mathrm{du} \mathrm{pH}$, cela est en accord avec les faibles valeurs en $\mathrm{HCO}_{3}{ }^{-}$enregistrées dans ces mêmes ouvrages et se traduit d'ailleurs par une bonne corrélation entre ces deux paramètres (Tableau 3). Ces résultats sont en accord avec ceux obtenus par Natchia et al. (2013) et Ahoussi et al. (2013) qui ont travaillé respectivement sur les eaux de la nappe d'altérites et les eaux de source de l'Ouest montagneux en Côte d'Ivoire. Des résultats similaires ont été aussi obtenus par Sandao (2013) dans les eaux du bassin de Korama de Zinder qui a montré que les pH très acides au niveau des nappes du Continental Hamadien et Terminal (grès ferrugineux), seraient dus essentiellement au processus géologique impliquant la diminution des ions bicarbonates et la libération des ions Hydrogènes avec précipitation de la sidérite. Ce qui est en faveur de notre hypothèse avancée ci-haut.

\section{Faciès hydrochimiques}

Les résultats issus de l'analyse hydrochimique ont permis de mettre en évidence quatre types de faciès avec une prédominance bicarbonatée sodique et potassique dans les eaux de la région de Zinder. De nombreux auteurs qui ont étudié l'hydrochimie en Afrique de l'Ouest ont abouti à la conclusion selon laquelle le faciès bicarbonaté est le plus abondant dans les eaux souterraines du CI/H et du CT (Lasm et al., 2011; Sandao, 2013 ; Amadou et al., 2014a ; Amadou et al., 2014b). Cette prédominance bicarbonatée-sodique peut se justifier par des teneurs importantes en bicarbonate et en sodium enregistrées dans les eaux de ces aquifères.

\section{Analyse en composantes principales}

$\mathrm{Au}$ regard des résultats des analyses statistiques de cette étude, les paramètres chimiques influençant la minéralisation des eaux de la région de Zinder selon la matrice de corrélation sont: $\mathrm{Ca}^{2+} \mathrm{Na}^{+}, \mathrm{SO}_{4}{ }^{2-}, \mathrm{HCO}_{3}{ }^{-}$, $\mathrm{Cl}^{-}$(Tableau 4). Ainsi, parmi les 14 paramètres utilisés pour l'analyse en composantes principales, trois ne participent pas à la constitution des trois axes factoriels : il s'agit des ions fluorures, des ions nitrites et de la température (Tableau 5). Cela pourrait être due à l'hétérogénéité des eaux de ces 
aquifères et des faibles valeurs de ces ions enregistrés, car selon les travaux de Mohemadou et al. (2008), sur les 19 paramètres qu'il a étudiés, 5 d'entre eux ne participent pas à la constitution des axes factoriels. En effet, il semble que deux principaux mécanismes gouvernent la minéralisation des eaux de la région de Zinder : le temps de séjour dans l'aquifère et le pluviolessivage des sols. La prédominance des ions hydrogénocarbonates enregistrées dans certains ouvrages pourrait provenir de l'altération de certains minéraux feldspathiques sous l'action de l'eau météorique plus ou moins chargée en $\mathrm{CO}_{2}$. Cette hypothèse peut être appuyée par une corrélation significative enregistre entre les ions $\mathrm{HCO}_{3}{ }^{-}$et les ions $\mathrm{Na}^{+}(0,830)$. Dans ce cas le $\mathrm{pH}$ de l'eau devient de plus en plus alcalin et la concentration des ions $\mathrm{HCO}_{3}{ }^{-}$va augmenter dès que le $\mathrm{CO}_{2}$ est disponible. Selon Njueya et al. (2012), les ions bicarbonates proviennent de l'altération de certains minéraux (feldspaths, calcite...), ce qui est en faveur de notre hypothèse avancée ci-haut. Donc les teneurs enregistrées en bicarbonate dans les eaux souterraines de ces aquifères renseignent sur le temps de séjour de l'eau dans l'aquifère (Lasm et al., 2011). Ces résultats nous permettent de dire que les teneurs en bicarbonates obtenues dans cette étude sont en accord avec la formation géologique de notre zone d'étude dont les principaux constituants géologiques sont les grès grossiers feldspathiques à ciment argileux dans le $\mathrm{C} / \mathrm{H}$ et des grès argileux et oolithes ferrugineux dans le CT. Les ions sodiums et potassiums pourraient provenir de l'altération de l'albite et de l'orthose qui vont libérer respectivement les ions bicarbonates et sodiums et les ions bicarbonates et potassiums (Njueya et al., 2012). Cependant, les résultats de nos analyses montrent que les ions sodiums sont prédominants par rapport aux ions potassiums dans les eaux de ces aquifères. En outre, selon la matrice de corrélation, il existe une bonne corrélation entre les ions sodiums et les ions bicarbonates, ce qui n'est pas le cas avec le potassium. Ceci pourrait être expliqué par la grande stabilité de la muscovite et des feldspaths potassiques d'une part et par l'adsorption des ions potassiums par les plantes d'autre part (Oga et al., 2009), ou à une grande sélectivité d'adsorption de potassium par les argiles. Cette hypothèse peut être appuyée par le fait qu'aucune corrélation n'existe entre ces deux éléments, donc ils peuvent évoluer différemment dans le milieu ce qui serait confirmé par les rapports $\mathrm{Na}^{+} / \mathrm{K}^{+}$qui sont supérieurs à 1 dans la majorité des ouvrages. L'abondance des ions calciums pourrait être due à l'infiltration des eaux des pluies à travers les minéraux renfermant le calcium tel que les plagioclases qui sont présents dans la formation géologique de notre zone d'étude tandis que le magnésium pourrait avoir trois origines dans les eaux souterraines : altération des minéraux ferromagnésiens, dissolution de la dolomite et substitution entre le magnésium libéré du talc et l'aluminium libéré (Njueya et al., 2012). Cependant, les résultats de notre étude, montrent qu'il y a une bonne corrélation entre le magnésium et le potassium, cela stipule que ces ions résultent principalement de l'altération des minéraux ferromagnésiens qui sont présents dans notre zone d'étude. Cela est corroboré par les résultats de Cheikh et al. (2011) qui ont montré que la dissolution du $\mathrm{Ca}^{2+}$ et $\mathrm{Mg}^{2+}$ résulte principalement de l'infiltration des eaux de surface à travers les formations rocheuses calcaires et dolomitique. La teneur relativement élevée en nitrates enregistrée dans l'ouvrage $\mathrm{PC}_{4}$, pourrait avoir principalement une origine anthropique. Mais, les ions nitrates présentent plusieurs origines parmi lesquelles: les roches, les apports météoriques, engrais, la décomposition de la végétation dans les sols et les polluants domestiques (Faillat in Yao, 2009). Cependant dans notre zone d'étude, les fortes teneurs en nitrates de $\mathrm{PC}_{4}$ pourraient être dues aux polluants domestiques, parce qu'il se trouve au centre des agglomérations et à côté du marché où l'insalubrité est très remarquable avec des déjections animales. Ce puits constitue aussi le point d'abreuvages des animaux le jour du marché et en plus il n'est pas équipé d'un système de protection. Donc la décomposition de ces matières pourrait 
augmenter les teneurs en nitrates de ce puits soit par infiltration des eaux de pluies ou par entraînement par les cordes utilisées pour puiser l'eau. Certains auteurs (Yao, 2009; Yameogo, 2008 ; Abdou Babayé et al., 2016) lient les teneurs élevées en nitrates aux activités anthropiques (production des déchets domestiques, réseau d'égout et latrines). $\mathrm{Ce}$ qui est en faveur de notre hypothèse cité cihaut.

\section{Conclusion}

La caractérisation des eaux des aquifères du $\mathrm{CI} / \mathrm{H}$ et du $\mathrm{CT}$ de la région de Zinder selon les résultats des analyses physico-chimiques, hydrochimiques (diagramme de Piper) et des analyses statistiques a permis de mettre en évidence les caractéristiques de ces eaux et leur potabilité potentielle. Dans l'ensemble, la plupart des valeurs obtenues dans cette étude sont conformes aux valeurs guides recommandées par l'OMS. Cependant, il existe, dans certains ouvrages, notamment ceux qui captent les aquifères du $\mathrm{CH}$ et du $\mathrm{CT}$, une certaine acidité des eaux dont les valeurs sont inférieurs à 6,5 et dans certains ouvrages qui captent le $\mathrm{CI} / \mathrm{H}$ des fortes teneurs en fluorures qui dépassent $1,5 \mathrm{mg} \cdot \mathrm{L}^{-1}$. Ces fortes teneurs en fluorures peuvent provoquer des maladies telles que la fluorose dentaire ou osseuse. Il est donc important de proposer des solutions pour ramener ces teneurs aux normes internationales des eaux de consommation. La classification des eaux selon le diagramme triangulaire de Piper a montré quatre type de faciès: les eaux bicarbonatées sodiques et potassiques $(53,57 \%)$, les eaux chlorurées sodiques et potassiques $(25 \%)$, les eaux chlorurées calciques et magnésiennes $(17,85$ $\%$ ) et les eaux bicarbonatées calciques et magnésiens (3,57 \%). L'analyse en composantes principales indique que la minéralisation des eaux étudiées est gouvernée par deux grands phénomènes: le temps de séjour et le pluviolessivage des sols. Les perspectives de nos travaux nous conduiraient à proposer des mécanismes simples et moins onéreux pour l'élimination du fluor dans les eaux étudiées par un 'mécanisme d'adsorption.

\section{CONFLIT D'INTERETS}

Les auteurs déclarent qu'ils n'ont pas d'intérêts concurrents

\section{CONTRIBUTIONS DES AUTEURS}

SMR a fait l'échantillonnage, les analyses et la rédaction du manuscrit; MAMM a coordonné l'étude et révision subséquente fondé sur les commentaires des examinateurs; MSL et NI ont participé à la rédaction du manuscrit; $\mathrm{IH}$ a fait certaines analyses.

\section{REMERCIEMENTS}

Nos remerciements vont à l'endroit des responsables de la Direction régionale de l'hydraulique et de l'assainissement de Zinder, pour la mise à notre disposition des équipements nécessaires pour la réalisation de ce travail.

\section{REFERENCES}

Njueya AK, Likeng JDH, Nono A. 2012. Hydrodynamique et qualité des eaux souterraines dans le bassin sédimentaire de Douala (Cameroun): cas des aquifères sur formations Quaternaires et Tertiaire. Int. J. Biol. Chem. Sci., 6(4): 1874-1894.

DOI: http://dx.doi.org/10.4314/ijbcs.v6i4.41

Ahoussi KE, Koffi YB, Kouassi AM, Soro G, Biemi J. 2013. Etude hydrochimique et microbiologique des eaux de source de l'ouest montagneux de la Côte d'Ivoire : cas du village de Mongouin-Yrongouin (sous-préfecture de Biankouma). Journal of Applied Biosciences, 63: 4703 - 4719.

Boubakar AH. 2010. Aquifères superficiels et Profonds et pollution urbaine en Afrique: cas de la communauté de Niamey (Niger). Thèse de doctorat, Université Abdou Moumouni de Niamey, Niger, p. 249.

Natchia A, Barthelemy BS, Gbombele S, Nagnin S. 2013. Etude hydrochimique et microbiologique des nappes d'altérites sous climat tropical humide: cas du 
department d'Abengourou (Sud-Est de la Côte d'Ivoire). Larhyss. Journal, 16: 3152.

Amadou H, Laouali MS, Manzola A. 2014. Analyse physicochimiques et bactériologiques des eaux de trios aquifères de la région de Tillabery : application des méthodes statistiques multivariées. Larhyss. Journal, 20: 2541.

Loukman B, Nakolendousse S, Nour AM, Memti MN. 2017. Caractérisation hydrochimique de la nappe de Yao et ses environs: relations entre eaux de surface (lac-Fitri) et eaux souterraines. Int. J. Biol. Chem. Sci., 11(3): 1336-1349. DOI : $\quad$ https://dx.doi.org/10.4314/ijbcs. v11i3.33

Mohamedou EO, Lebkiri A, Rifi EH, Lebkiri M, Fadli M, Pointie M, Mahmoud AKO,Fagel ML. 2008. Typologie physico-chimique et métallique des eaux du fleuve Sénégal au niveau de la ville de Rosso (Mauritani). Afrique. Science, 04(3): 394-409.

Malcuit E. 2012. Origine de la minéralisation des eaux dans un aquifère multicouche profond - Exemple de la "zone minéralisée de l'Entre-Deux-Mers"' (Bassin aquitain, France). Thèse de doctorat, Université Michel de Montaigne Bordeaux 3. p137.

Ahoussi EK, Soro N, Kouassi AM, Soro G, Koffi YB, Zade SP. 2010. Application des methods d'analyses statistiques multivariées à l'étude de l'origine des métaux lourds $\left(\mathrm{Cu}^{2+}, \mathrm{Mn}^{2+}, \mathrm{Zn}^{2+}\right.$ et $\left.\mathrm{Pb}^{2+}\right)$ dans les eaux des nappes phréatiques de la ville d'Abidjan. Int. J. Biol. Chem. Sci., 4(5): 1753-1765.

Chemseddine F, Abderrahmane B, Abdelkader R, Elias S. 2009. Caractérisation hydrogéochimique des eaux souterraines du complexe aquifère Morsott-Laouinet (Région Nord de Tébessa, Sud-Est algérien. Afrique Science, 05(2): 217-231.

Abdou Babayé MS, Sandao I, Saley MB, Wagani I, Ousmane B. 2016. Comportement hydrogéochimique et contamination des eaux des aquifères fissurés du socle précambrien en milieu semi-aride (Sud-Ouest du Niger). Int. J. Biol. Chem. Sci., 10(6): 2728-2743. DOI: http://dx.doi.org/10.4314/ijbcs.v10i6.26

Amadou H, Laouali MS, Manzola AS. 2014. Application des méthodes d'analyses statistiques multivariées à l'étude de la minéralisation des eaux de la zone de Zinder (Sud-Est du Niger). Int. J. Biol. Chem. Sci., 8(4): 1904-1916. DOI: http://dx.doi.org/10.4314/ijbcs.v8i4.50

Amadou H, Laouli MS, Manzola AS. 2014. Caractérisation hydro chimique des eaux souterraines de la région de Tahoua (Niger). Journal of Applied Bioscience, 80: $\quad 7161-7172 . \quad$ DOI: http://dx.doi.org/10.4314/jab.v81i1.6

Rodier J, Legube B, Merlet N. 2009. L'Analyse de l'Eau (9 edn). DUNOD: Paris.

Ravindra K, Garg VK. 2007. Hydrochemical Survey of Defluoridation Methods used in India. Environ Monit Assess, 132: 3343. DOI 10.1007/s10661-006-9500-6

Oga M-S, Lasm T, Yao TK, Soro N, Saley MB, Kouassi D, Gnamba F. 2009. Caractérisation chimique des eaux des Aquifères de Fracture : cas de la Région de Tiassalé en Côte d'Ivoire. Europen Journal of Scientific Research, 31(1): 72-87. DOI: http://www.eurojournals. com/ejsr.htm

Matini L, Moutou JM, KONGO-MANTON MS. 2009. Evaluation hydro-chimique des eaux souterraines en milieu urbain au Sud-Ouest de Brazzaville. Afrique Science, 05(1): 82-98.

Kouassi MA, Yao AK, Ahoussi EK, Seki CL, Yao AN, Kouassi IK, Biemi J. 2010. Apport des méthodes statistiques et hydrochimiques à la caractérisation des eaux des aquifères fissurés de la région du N'zi-Comoé (centre-Est de la Côte d'Ivoire). Int. J. Biol. Chem. Sci., 4(5): 1816-1838.

Cheikh MEKO, Kacemi KE, Idrissi L. 2011. Caractérisation physico-chimique des eaux d'alimentation de la ville de Tijikja (mauritani). Int. J. Biol. Chem. Sci., 5(5): 
2133-2139. DOI: http://dx.doi.org/10. 4314/ijbcs.v5i5.33

Yameogo SO. 2008. Ressources en eau souterraine du centre urbain de Ouagadougou au Burkina Faso qualité et vulnérabilité. Thèse de doctorat, Université d'Avignon et des Pays de Vaucluse, p. 254.

OMS. 2011. Guildelines for Drinking-Water Quality ( $4^{\text {th }}$ edn). OMS: Geneva.

Issa S, Mama D, Zanguina A, Natatou I, Boukari M, Sohounhloué D. 2015. Hydrogeochemical characterization of Continental Intercalary Terminal, the base and the Korama aquifers groundwater found in five local Governments in the Zinder region in the Republic of Niger. Research Journal of Chemical and Environmental Sciences, 3(4): 09-16.

Sandao I. 2011. Etudes hydrodynamique, hydrochimique et isotopique des eaux souterraines du bassin versant de la Korama / Sud Zinder, Niger : impacts de la variabilité climatique et des activités anthropiques. Thèse de doctorat, Université Abdou Moumouni de Niamey, Niger, p. 211.

Sridhar N, Chandrasekarn N, Subbarayan MR. 2016. High fluoride concentration in the groundwater of Uttangari Taluk, Krishnagiri District, South of India. Int. J. Inn. Res. Sci. Eng., 24(2): 200-210

Tabouche N, Achour S. 2004. Etude de la qualité des eaux souterraines de la région orientale $\mathrm{du}$ Sahara septentrional Algérien. Larhyss. Journal, 3: 99-113.

Lasm T, Lasme OD, Oga M-SY, Younata M, Baka D, Kouame F, Yao TK. 2011. Caractérisation hydrochimique des aquifères fissurés de la région de SanPedro (Sud-Ouest de la Côte d'Ivoire). Int. J. Biol. Chem. Sci., 5(2): 642-662.

Yao TK, OGA M-S, Fouché O, Baka D, Pernelle C, Biemi J. 2012. Evaluation de la qualité chimique des eaux souterraines dans un bassin versant tropical : cas du Sud-Ouest de la Côte d'Ivoire. Int. J. Biol. Chem. Sci., 6(6): 7069-7086. DOI: http://dx.doi.org/10.4314/ijbcs.v6i6.42

Yao TK. 2009. Hydrodynamisme dans les aquifères de socle cristallin et cristallophyllien du sud-ouest de la Côte d'Ivoire : cas du département de Soubré. Apports de la télédétection, de la géomorphologie et de l'hydrogéochimie. Thèse de doctorat, Université Cocody, Côte d'Ivoire, p. 337. 